\title{
BREAST SURGERY
}

\section{Testing the feasibility of intra-operative sentinel lymph node touch imprint cytology}

\author{
ALIREZA HAMIDIAN JAHROMI ${ }^{1}$, SANKARAN NARAYANAN ${ }^{2}$, FIONA MaCNEILL ${ }^{2}$, PETER OSIN ${ }^{3}$, \\ ASHUTASH NERURKAR ${ }^{3}$, GERALD GUI ${ }^{2}$
}

\author{
${ }^{1}$ Renal Transplant Department, St George's Hospital, London, UK \\ Departments of ${ }^{2}$ Breast Surgery and ${ }^{3}$ Histopathology, The Royal Marsden NHS Foundation Trust, \\ London, UK
}

ABSTRACT

INTRODUCTION Sentinel lymph node biopsy is emerging as the new standard for axillary staging in breast cancer. Intra-operative assessment of the sentinel lymph nodes allows immediate completion of axillary dissection during the same anaesthetic. This project was a quality assurance practice to establish feasibility, time-to-report, as well as accuracy of performing intraoperative assessment of sentinel lymph nodes using touch imprint cytology in our centre.

PATIENTS AND METHODS This prospective audit included 146 sentinel lymph nodes from 74 consecutive patients with invasive breast cancer. All patients underwent axillary sentinel lymph node biopsy using combined blue dye and radiocolloid technique. Results of intra-operative touch imprint cytology using haematoxylin and eosin staining were compared with the definitive histopathology results.

RESULTS Mean time to report touch imprint cytology was $25.7 \pm 6.4$ min (range, 15-40 min). Histopathology demonstrated metastasis in 25 sentinel nodes from 17 (23\%) patients. Intra-operative touch imprint cytology detected 15 nodes in 11 patients, giving a sensitivity of $60 \%$ (nodes) and $66.7 \%$ (patients) and specificity of $99.2 \%$ (nodes) and $98.2 \%$ (patients) based on the number of nodes and patients involved, respectively. Touch imprint cytology failed to show metastatic involvement in 10 nodes from 6 patients; of these, five nodes had micrometastasis $(<2 \mathrm{~mm})$ and the other five had macrometastasis. One touch imprint cytology positive node contained isolated tumour cells only. Using intra-operative touch imprint cytology made a change in treatment of $11(14.9 \%)$ patients, and spared second axillary procedure in 7 (9.4\%) patients.

CONCLUSIONS Intra-operative sentinel lymph node assessment using touch imprint cytology is feasible within a busy NHS practice. We now offer touch imprint cytology to patients following appropriate counselling.

KEYWORDS

Touch imprint cytology - Sentinel lymph node - Breast cancer

CORRESPONDENCE TO

Alireza Hamidian Jahromi, Renal Transplant Department, St George's Hospital, London SW17 OQT, UK

E: alirezahamidian@yahoo.com

Sentinel lymph node biopsy (SLNB) is replacing routine axillary dissection and is the new standard for axillary staging in early breast cancer supported by some uni-centre and multi-centre randomised controlled trials. ${ }^{1,2}$ Sentinel lymph nodes are generally evaluated postoperatively, requiring 5-7 days for pathological assessment. Axillary clearance remains standard treatment for patients with axillary metastases identified on sentinel lymph node biopsy. ${ }^{3}$

Intra-operative assessment of the sentinel lymph nodes allows immediate and therapeutic completion of axillary dissection during the same anaesthetic session if nodes are found to be tumour-positive. ${ }^{4}$ In addition, delayed axillary clearance after sentinel lymph node biopsy can be technically challenging with the potential risk of increased morbidity (particularly infection and seroma) and lower overall nodal yield. Intra-operative touch imprint cytology (TIC) will also help by avoiding the economic and psychological costs of a second operation and prevents further delay in the commencement of adjuvant systemic treatment, chemotherapy and radiotherapy for high-risk patients with confirmed node involvement. ${ }^{5}$

Frozen-section examination and TIC are two methods used for intra-operative evaluation of sentinel lymph nodes. Although frozen section examination is more expensive than TIC and has disadvantages (i.e. it is more difficult to perform, requires more time for processing and is associated with tissue loss, which might affect further histologial examinations) it is still more commonly used than TIC. ${ }^{6}$ 
Table 1 Touch imprint cytology versus histopathology results based on the number of the nodes and patients studied

\begin{tabular}{lcccc} 
& \multicolumn{2}{c}{ Number of nodes } & \multicolumn{2}{c}{ Number of patients } \\
& TIC positive & TIC negative & TIC positive & TIC negative \\
Histopathology positive & $15(10.3 \%)$ & $10(6.8 \%)$ & $11(14.9 \%)$ & $6(8.1 \%)$ \\
Histopathology negative & $1(0.7 \%)$ & $120(82.2 \%)$ & $1(1.3 \%)$ & $56(75.7 \%)$
\end{tabular}

TIC, touch imprint cytology.

Touch imprint cytology is quicker to perform, requires minimal tissue preparation, can be done using simple equipment and will provide acceptable details for interpretation. However, it requires experienced cytopathologists.

This is a quality assurance audit to establish overall feasibility, time-to-report, as well as accuracy of performing intra-operative assessment of sentinel lymph nodes using TIC in our centre.

\section{Patients and Methods}

This prospective audit included 74 patients with invasive breast cancer, who were treated in the Royal Marsden Hospital in London, UK between May 2006 and February 2007. All patients had pre-operative diagnosis of invasive breast carcinoma with clinically normal and image-negative axillae. They underwent axillary sentinel lymph node biopsy using combined technique of blue dye (Patent Bleu V; Guerbet Laboratories, Issy Les Moulineaux, France) and radiocolloid identification ( ${ }^{99 \mathrm{~m}} \mathrm{Tc}-\mathrm{Lab}$ lled Nanocoll; Amersham Health, Little Chalfont, UK), with routine preoperative sentinel node imaging.

A sentinel lymph node was defined as any blue or hot node with a total count of $>10 \%$ of the highest node count. Sentinel lymph node biopsy was performed before removal

Table 2 Sensitivity, specificity, positive predictive value (PPV) and negative predictive value (NPV) based on the number of sentinel nodes and patients studied
Based on number of patients

Based on number of nodes

Sensitivity $\quad 66.7 \%(0.43-0.83)$

Specificity $\quad 98.2 \%(0.90-0.99)$

PPV

NPV
$60.0 \%(0.40-0.76)$

$99.2 \%(0.95-1.00)$

$93.8 \%(0.71-0.99)$

$92.3 \%(0.86-0.95)$
Figures in parenthesis are $95 \%$ confidence intervals. of the primary tumour. Intra-operative TIC using standard hematoxylin and eosin staining of the freshly taken sentinel nodes were performed for all patients.

Sentinel nodes were bivalved along their long axis and imprinted directly on to coated microscopy slides. At least two imprints from each bivalved node were made on a separate microscope slide. Sentinel lymph node removal-toreport time was recorded in all patients. Indeterminate or suspicious TIC results were considered as negative results.

Results of the TIC were compared with the definitive postoperative histopathology results. Postoperative histopathology assessment included hematoxylin and eosin staining for all of the specimens. Immunohistochemical staining was used for equivocal results.

\section{Results}

In this study, 146 nodes were examined from 74 patients with invasive breast cancer (average node yield, 2 nodes per patient). Mean age of the patients was $49.59 \pm 13.6$ years (range, 23-78 years) and mean time-to-report TIC was 25.7 $\pm 6.4 \mathrm{~min}$ (range, 15-40 min). Touch imprint cytology and postoperative histopathology results are shown for number of nodes and patients in Table 1.

Postoperative histopathology results demonstrated metastasis within 25 sentinel lymph nodes from 17 (23\%) patients. Seventeen nodes showed macrometastasis (> $2 \mathrm{~mm}$ ) and eight nodes showed micrometastasis involvement $(<2 \mathrm{~mm})$. Intra-operative TIC detected 15 of the involved nodes in 11 patients, giving this technique sensitivity of $60 \%$ and $66.7 \%$ and specificity of $99.2 \%$ and $98.2 \%$ based on the number of lymph nodes and patients involved, respectively.

Sensitivity, specificity, positive predictive value and negative predictive value have been calculated separately based on the number of the sentinel lymph nodes and patients and are summarised in Table 2 .

Intra-operative TIC failed to show metastatic involvement in 10 examined lymph nodes from six patients; of these, 5 nodes had micrometastasis $(<2 \mathrm{~mm})$ and the other 5 had macrometastasis $(>2 \mathrm{~mm})$ on postoperative hema- 
toxylin and eosin analysis. One TIC positive node (the only sentinel lymph node removed for that patient) contained isolated tumour cells only.

\section{Discussion}

Axillary status has been proven to be the strongest prognostic factor for women with early breast cancer. ${ }^{7,8}$ Accurate staging has traditionally been accomplished with a Level I and II axillary lymph node dissection (ALND).

Although ALND provides good prognostic information as well as local control in patients with metastatic axillary disease, morbidity can be high (e.g. lymphoedema, loss of function, etc.). Consequently, less invasive staging with lower morbidity risk (sentinel lymph node biopsy) has been promoted.

Multiple trials have documented that lympathic mapping and subsequent SLNB reflects the stage of axillae. ${ }^{9-11}$ However, the extent to which SLNB provides local control is currently unknown. Therefore, completion ALND is still indicated for patients with a node-positive specimen. ${ }^{3}$

Intra-operative assessments of the sentinel lymph nodes to ascertain lymphatic metastasis allows immediate completion of ALND during the same operative session. Different advantages of intra-operative assessments of the sentinel nodes include: (i) avoiding the technical challenge of ALND after sentinel lymph node biopsy; (ii) its increased morbidity risk; (iii) avoiding economic and psychological costs of a second operation; and (iv) preventing further delay in systemic treatment and radiotherapy.

However, it has its own disadvantages as well: the risk of over treatment, uncertainty for patient regarding outcome of surgery, difficulties in consenting the patients, planning theatre lists, in-patient beds, and its requirement for availability of cytopathological expertise.

Many strategies have been investigated for intra-operative assessment of the sentinel nodes including extensive evaluation of intra-operative frozen sections, intra-operative TIC and intra-operative TIC with the adjunct of immediate immunohistochemical staining for cytokeratin. Different studies have compared these techniques with regard to the following three considerations - speed (required time-to-report), reliability and cost-effectiveness. ${ }^{12,13}$

In our study, time-to-report TIC fitted comfortably with average times for completion of breast surgery. Although the average time taken for reporting TIC appears acceptable, the additional anaesthetic time in a minority of patients must be offset against the increased operating time involved in a delayed axillary clearance.

Accuracy of TIC in predicting node involvements is 'operator dependent' as reflected in very variable range of reported accuracy for this method in different studies. ${ }^{6}$ Reported sensitivity of TIC varies from 33.3 to $100 \% .^{14-16}$
Overall sensitivity and specificity of TIC in the present study based on the number of the patients was $66.7 \%$ and $98.2 \%$, respectively. In this study, the pressure of immedate intraoperative reporting and the fact that the plan for continuation of surgical procedure would be based on the report, did not affect the accuray. Our TIC accuracy was consistent with other studies. ${ }^{6,17}$

Sensitivity of TIC in our practice may be affected by preselection of gross node-positive patients by axillary ultrasound. In these situations, we normally proceed with ALND directly. All of our patients had pre-operative clinically normal and image-negative axillae. Touch imprint cytology sensitivity in unselected patients would be much higher.

Many of our patients have early disease so the burden of nodal disease is small and, therefore, less amenable to TIC. Intra-operative TIC failed to show metastatic involvement in 10 examined lymph nodes from 6 patients; of these, 5 nodes had micrometastasis $(<2 \mathrm{~mm})$ on postoperative hematoxylin and eosin analysis.

Touch imprint cytology sensitivity is dependent on the burden of disease within a node. In early disease, the nodal metastatic cell count is, therefore, relatively 'low' demonstrating the limitations of TIC. Hence, in these situations, there is a drive towards more sensitive techniques of nodal analysis such as polymerase chain reaction techniques. ${ }^{18,19}$

A false-positive intra-operative TIC would make the surgeon proceed with ALND while it might not be necessary, thereby the initial intention of SLNB to decrease morbidity caused by ALND would not be achieved. ${ }^{6}$ In the current study, indeterminate or suspicious TIC results were considered as negative to maintain high specificity and avoid unnecessary ALND.

In our practice, pre-operative axillary scanning selects out gross node-positive patients so the benefits of TIC in reducing the number of second operations is potentially limited. Using intra-operative TIC changed treatment in 11 $(14.9 \%)$ patients, and spared second axillary procedure in seven $(9.4 \%)$ patients. The remaining four patients still required another surgical intervention for positive margins. However, they proceeded with axillary clearance at an earlier time and in the setting of their initial operation. One patient had an ALND for isolated tumour cells.

\section{Conclusions}

Intra-operative sentinel lymph node assessment using TIC is feasible within a busy NHS practice but it only changed management in $15 \%$ of our patients. Therefore, its application needs careful consideration. We now offer TIC to suitably counselled patients. Patients predicted to have significant risk of node-positive axillae, considering pre-operative core biopsy results and tumour size, patients with high risk of anaesthesia (American Society of Anesthesiologists [ASA] Class 
$>$ III) and patients undergoing mastectomy who are not likely to require further breast surgery are examples of patients who would benefit more from intra-operative TIC.

\section{Acknowledgements}

The authors thank Ms Sylvia Tan, audit co-ordinator and Ms Sue Ashley, consultant statistician, for their kind support in analyzing the data of this study from the Royal Marsden NHS Foundation Trust, London, UK.

\section{References}

1. Veronesi U, Paganelli G, Viale G, Luini A, Zurrida S, Galimberti V et al. A randomized comparison of sentinel node biopsy with routine axillary dissection in breast cancer. N Engl J Med 2003; 394: 546-53.

2. Mansel RE, Fallowfield L, Kissin M, Goyal A, Newcombe RG, Dixon JM et al. Randomized multicenter trial of sentinel node biopsy versus standard axillary treatment in operable breast cancer: the ALMANAC Trial. J Natl Cancer Inst 2006; 98: 599-609.

3. Lyman GH, Giuliano AE, Somerfield MR, Benson 3rd AB, Bodurka DC, Burstein $\mathrm{HJ}$ et al. American Society of Clinical Oncology guideline recommendations for sentinel lymph node biopsy in early-stage breast cancer. J Clin Oncol 2005; 23 7703-20.

4. Pugliese MS, Kohr JR, Allison KH, Wang NP, Tickman RJ, Beatty JD. Accuracy of intraoperative imprint cytology of sentinel lymph nodes in breast cancer. Am J Surg 2006; 192: 516-9.

5. Jeruss JS, Hunt KK, Xing Y, Krishnamurhy S, Meric-Bernstam F, Cantor SB et al. Is intraoperative touch imprint cytology of sentinel lymph nodes in patients with breast cancer cost effective? Cancer 2006; 107: 2328-36.

6. Chicken DW, Kocjan G, Falzon M, Lee AC, Douek M, Sainsbury R et al. Intraoperative touch imprint cytology for the diagnosis of sentinel lymph node metastases in breast cancer. Br J Surg 2006; 93: 572-6.

7. Fitzgibbons PL, Page DL, Weaver D, Thro AD, Allred DC, Clark GM et al.
Prognostic factors in breast cancer. College of American Pathologists Consensus Statement 1999. Arch Pathol Lab Med 2000; 124: 966-78.

8. Singletary SE, Allred C, Ashley P, Bassett LW, Berry D, Bland KI et al. Revision of the American Joint Committee on Cancer staging system for breast cancer. $J$ Clin Oncol 2002; 20: 3628-36.

9. Giuliano AE, Kirgan DM, Guenther JM, Morton DL. Lymphatic mapping and sentinel lymphadenectomy for breast cancer. Ann Surg 1994; 220: 391-8.

10. Albertini JJ, Lyman GH, Cox C, Yeatman T, Balducci L, Ku N et al. Lymphatic mapping and sentinel node biopsy in the patient with breast cancer. JAMA 1996; 276: 1818-22.

11. Kelley MC, Hansen N, McMasters KM. Lymphatic mapping and sentinel lymphadenectomy for breast cancer. Am J Surg 2004; 188: 49-61.

12. Cox C, Centeno B, Dickson D, Clark J, Nicosia S, Dupont E et al. Accuracy of intra-operative imprint cytology for sentinel lymph node evaluation in the treatment of breast carcinoma. Cancer 2005; 105: 13-20.

13. Aihara T, Munakata S, Morino H, Takatsuka Y. Comparison of frozen section and touch imprint cytology for evaluation of sentinel lymph node metastasis in breast cancer. Ann Surg Oncol 2004; 11: 747-50.

14. Barranger E, Antoine M, Grahek D, Callard P, Uzan S. Intra-operative imprint cytology of sentinel nodes in breast cancer. J Surg Oncol 2004; 86: 128-33.

15. Usman T, Rowlands DC, England DW. Rapid preoperative assessment of axillary lymph node status using imprint cytology. Breast 1999; 8: 101-3.

16. Perez N, Vidal-Sicart S, Zanon G, Velasco M, Santamaria G, Palacin A et al. A practical approach to intra-operative evaluation of sentinel lymph node biopsy in breast carcinoma and review of the current methods. Ann Surg Oncol 2005; 12: 313-21.

17. Tew K, Irwig L, Matthews A, Crowe P, Macaskill P. Meta-analysis of sentinel node imprint cytology in breast cancer. Br J Surg 2005; 92: 1068-80.

18. Treseler P. Pathologic examination of the sentinel lymph node: what is the best method? Breast J 2006; 12 (Suppl 2): S143-51.

19. Julian TB, Blumencranz P, Deck K, Whitworth P, Berry DA, Berry SM et al. Novel intraoperative molecular test for sentinel lymph node metastases in patients with early-stage breast cancer. J Clin Oncol 2008; 26: 3338-45.

\section{Erratum}

Ann $R$ Coll Surg Engl 2009; 91: 81-82

A new technique for ensuring local anaesthesia in tunnelled central venous catheters Ashok Daya Ram, Robert A Wheeler

We apologise for the incorrect spelling of Mr Ram's middle name in the above Technical Note.

The correct name is Daya, as given above. 